\title{
Blow-up Solutions of Quasilinear Hyperbolic Equations With Critical Sobolev Exponent
}

\author{
S. Ibrahim ${ }^{1 *}$, A. Lyaghfouri ${ }^{2}$ \\ ${ }^{1}$ Department of Mathematics and Statistics, University of Victoria, Victoria, BC, Canada \\ ${ }^{2}$ Fields Institute, Toronto, ON, Canada
}

\begin{abstract}
In this paper, we show finite time blow-up of solutions of the $p$-wave equation in $\mathbb{R}^{N}$, with critical Sobolev exponent. Our work extends a result by Galaktionov and Pohozaev [4]
\end{abstract}

Keywords and phrases: $p$-wave equation, Blow-up, critical Sobolev exponent

Mathematics Subject Classification: 35L05, 35L70, 35L80

\section{Introduction}

In this paper, we consider the following $p$-wave equation:

$$
\left\{\begin{array}{l}
u_{t t}-\Delta_{p} u=|u|^{p_{*}-2} u \quad \text { in } \mathbb{R}^{N} \times \mathbb{R} \\
u(., 0)=u_{0} \in W^{1, p}\left(\mathbb{R}^{N}\right) \\
u_{t}(., 0)=u_{1} \in L^{2}\left(\mathbb{R}^{N}\right),
\end{array}\right.
$$

where $N \geq 3,2<p<N, \Delta_{p} u=\operatorname{div}\left(|\nabla u|^{p-2} \nabla u\right)$ is the $p$-Laplacian and $p_{*}=\frac{p N}{N-p}$ is the critical Sobolev exponent, and where

$$
\dot{W}^{1, p}\left(\mathbb{R}^{N}\right)=\left\{u \in L^{p_{*}}\left(\mathbb{R}^{N}\right): \nabla u \in L^{p}\left(\mathbb{R}^{N}\right)\right\} .
$$

Multiplying equation (1.1) by $u_{t}$ and integrating by parts, we formally get

$$
\int_{\mathbb{R}^{N}} u_{t} u_{t t} d x+\int_{\mathbb{R}^{N}}|\nabla u|^{p-2} \nabla u \cdot \nabla u_{t} d x-\int_{\mathbb{R}^{N}}|u|^{p_{*}-2} u u_{t} d x=0
$$

or

$$
\frac{d}{d t}\left(\frac{1}{2} \int_{\mathbb{R}^{N}}\left|u_{t}\right|^{2} d x+\frac{1}{p} \int_{\mathbb{R}^{N}}|\nabla u|^{p} d x-\frac{1}{p_{*}} \int_{\mathbb{R}^{N}}|u|^{p_{*}} d x\right)=0 .
$$

Setting $E(u(., t))=\frac{1}{2} \int_{\mathbb{R}^{N}}\left|u_{t}\right|^{2} d x+\frac{1}{p} \int_{\mathbb{R}^{N}}|\nabla u|^{p} d x-\frac{1}{p_{*}} \int_{\mathbb{R}^{N}}|u|^{p_{*}} d x$, we obtain

$$
\frac{d E(u(., t))}{d t}=0
$$

*Corresponding author. E-mail: ibrahims@uvic.ca 
In particular, we have

$$
E(u(., t))=E(u(., 0))=E\left(u_{0}, u_{1}\right)=\frac{1}{2} \int_{\mathbb{R}^{N}}\left|u_{1}\right|^{2} d x+\frac{1}{p} \int_{\mathbb{R}^{N}}\left|\nabla u_{0}\right|^{p} d x-\frac{1}{p_{*}} \int_{\mathbb{R}^{N}}\left|u_{0}\right|^{p_{*}} d x .
$$

Recall that the function

$$
w_{p}(x)=\left(1+\frac{p-1}{(N-p) N^{\frac{1}{p-1}}}|x|^{\frac{p}{p-1}}\right)^{\frac{p-N}{p}}
$$

solves the equation

$$
\Delta_{p} u=-|u|^{p_{*}-2} u \text { in } \mathbb{R}^{N}
$$

and is known as the ground state. It is related to the best constant of the Sobolev inequality

$$
\|u\|_{p_{*}} \leq C(N, p)\|\nabla u\|_{p} \quad \forall u \in \dot{W}^{1, p}\left(\mathbb{R}^{N}\right),
$$

where $C(N, p)$ is characterized by

$$
\left\{\begin{array}{l}
\text { if }\|u\|_{p_{*}}=C(N, p)\|\nabla u\|_{p} \text { and } u \neq 0, \text { then } \\
u=C w_{p}\left(\sigma\left(x-x_{0}\right)\right) \text { for some constants } C \neq 0, \sigma>0, \text { and } x_{0} \in \mathbb{R}^{N} .
\end{array}\right.
$$

For more details, we refer for example to [2], [10] and [1].

Define

$$
\mathcal{E}(u)=\frac{1}{p} \int_{\mathbb{R}^{N}}|\nabla u|^{p} d x-\frac{1}{p_{*}} \int_{\mathbb{R}^{N}}|u|^{p_{*}} d x
$$

and

$$
\mathcal{K}(u)=\int_{\mathbb{R}^{N}}|\nabla u|^{p} d x-\int_{\mathbb{R}^{N}}|u|^{p_{*}} d x
$$

One can easily derive the following identities satisfied by the ground state.

\section{Proposition 1.1.}

$$
\begin{aligned}
& \int_{\mathbb{R}^{N}}\left|w_{p}\right|^{p_{*}} d x=\int_{\mathbb{R}^{N}}\left|\nabla w_{p}\right|^{p} d x=\frac{1}{C^{N}(N, p)} \\
& \text { and } \mathcal{E}_{p}=\mathcal{E}\left(w_{p}\right)=\frac{1}{N} \int_{\mathbb{R}^{N}}\left|\nabla w_{p}\right|^{p} d x=\frac{1}{N C^{N}(N, p)}
\end{aligned}
$$

Proof. i) Multiplying equation (1.3) by $u$ and integrating over $\mathbb{R}^{N}$, we get

$$
\int_{\mathbb{R}^{N}} \Delta_{p} w_{p} \cdot w_{p} d x=-\int_{\mathbb{R}^{N}}\left|w_{p}\right|^{p_{*}} d x
$$

or

$$
\int_{\mathbb{R}^{N}}\left|\nabla w_{p}\right|^{p} d x=\int_{\mathbb{R}^{N}}\left|w_{p}\right|^{p_{*}} d x
$$

It follows that

$$
\left\|w_{p}\right\|_{p_{*}}=\left\|\nabla w_{p}\right\|_{p}^{\frac{p}{p_{*}}}=\left\|\nabla w_{p}\right\|_{p}^{\frac{p}{p_{*}}-1}\left\|\nabla w_{p}\right\|_{p}=\left\|\nabla w_{p}\right\|_{p}^{-\frac{p}{N}}|| \nabla w_{p}\left\|_{p}=\right\| w_{p}\left\|_{p_{*}}^{-\frac{p_{*}}{N}}\right\| \nabla w_{p} \|_{p} .
$$


We deduce that $C(N, p)=\left\|w_{p}\right\|_{p_{*}}^{-\frac{p_{*}}{N}}$, and we obtain from (1.8)

$$
\int_{\mathbb{R}^{N}}\left|\nabla w_{p}\right|^{p} d x=\int_{\mathbb{R}^{N}}\left|w_{p}\right|^{p_{*}} d x=|| w_{p} \|_{p_{*}}^{p_{*}}=\frac{1}{C^{N}(N, p)} .
$$

ii) Using (1.8), we get

$$
\begin{aligned}
\mathcal{E}_{p} & =\frac{1}{p} \int_{\mathbb{R}^{N}}\left|\nabla w_{p}\right|^{p} d x-\frac{1}{p_{*}} \int_{\mathbb{R}^{N}}\left|w_{p}\right|^{p_{*}} d x \\
& =\left(\frac{1}{p}-\frac{1}{p_{*}}\right) \int_{\mathbb{R}^{N}}\left|w_{p}\right|^{p_{*}} d x \\
& =\frac{1}{N} \int_{\mathbb{R}^{N}}\left|w_{p}\right|^{p_{*}} d x \\
& =\frac{1}{N C^{N}(N, p)} .
\end{aligned}
$$

Now we prove the following variational characterization of $w_{p}$.

\section{Proposition 1.2.}

$$
\mathcal{E}_{p}=\inf \left\{\mathcal{E}(u): u \in \dot{W}^{1, p}\left(\mathbb{R}^{N}\right), \mathcal{K}(u)=0, u \neq 0\right\} .
$$

Proof. First, by (1.6) we have $K\left(w_{p}\right)=0$, and therefore

$$
\inf \left\{\mathcal{E}(u): u \in \dot{W}^{1, p}\left(\mathbb{R}^{N}\right), \mathcal{K}(u)=0, u \neq 0\right\} \leq \mathcal{E}_{p} .
$$

Second, recall [2], [10] and [1] that

$$
\begin{gathered}
\left\|\nabla w_{p}\right\|_{p}^{p}=\inf \left\{\|\nabla u\|_{p}^{p}: u \in \dot{W}^{1, p}\left(\mathbb{R}^{N}\right),\|u\|_{p_{*}}=\left\|w_{p}\right\|_{p_{*}}\right\} \\
\left\|w_{p}\right\|_{p_{*}}^{p_{*}}=\sup \left\{\|u\|_{p_{*}}^{p_{*}}: u \in \dot{W}^{1, p}\left(\mathbb{R}^{N}\right),\|u\|_{p_{*}}=\left\|w_{p}\right\|_{p_{*}}\right\} .
\end{gathered}
$$

Now let $u \in \dot{W}^{1, p}\left(\mathbb{R}^{N}\right) \backslash\{0\}$ be such that $\mathcal{K}(u)=0$, and set $\widetilde{u}=\frac{\left\|w_{p}\right\|_{p_{*}}}{\|u\|_{p_{*}}} u$. Since $\|\widetilde{u}\|_{p_{*}}=\left\|w_{p}\right\|_{p_{*}}$, we have by (1.10)

$$
\begin{aligned}
& \left\|\nabla w_{p}\right\|_{p}^{p} \leq\|\nabla \widetilde{u}\|_{p}^{p}=\frac{\left\|w_{p}\right\|_{p_{*}}^{p}}{\|u\|_{p_{*}}^{p}}\|\nabla u\|_{p}^{p} \\
& \text { or } \frac{\left\|\nabla w_{p}\right\|_{p}^{p}}{\left\|w_{p}\right\|_{p_{*}}^{p}} \leq \frac{\|\nabla u\|_{p}^{p}}{\|u\|_{p_{*}}^{p}} .
\end{aligned}
$$

Since $\mathcal{K}(u)=\mathcal{K}\left(w_{p}\right)=0$, we have $\left\|\nabla w_{p}\right\|_{p}^{p}=\left\|w_{p}\right\|_{p_{*}}^{p_{*}}$ and $\|\nabla u\|_{p}^{p}=\|u\|_{p_{*}}^{p_{*}}$. We obtain from (1.12)

$$
\left\|w_{p}\right\|_{p_{*}}^{p_{*}-p} \leq\|u\|_{p_{*}}^{p_{*}-p}
$$

From Proposition 1.1, we know that

$$
\mathcal{E}_{p}=\frac{1}{N}\left\|w_{p}\right\|_{p_{*}}^{p_{*}}
$$


Moreover, since $\mathcal{K}(u)=0$, we have

$$
\begin{aligned}
\mathcal{E}(u) & =\frac{1}{p} \int_{\mathbb{R}^{N}}|\nabla u|^{p} d x-\frac{1}{p_{*}} \int_{\mathbb{R}^{N}}|u|^{p_{*}} d x \\
& =\frac{1}{p} \mathcal{K}(u)+\left(\frac{1}{p}-\frac{1}{p_{*}}\right) \int_{\mathbb{R}^{N}}|u|^{p_{*}} d x \\
& =\left(\frac{1}{p}-\frac{1}{p_{*}}\right) \int_{\mathbb{R}^{N}}|u|^{p_{*}} d x \\
& =\frac{1}{N}\|u\|_{p_{*}}^{p_{*}}
\end{aligned}
$$

We deduce from (1.13)-(1.15) and since $p_{*}>p$, that

$$
\begin{aligned}
& \left(N \mathcal{E}_{p}\right)^{\frac{p_{*}-p}{p_{*}}} \leq(N \mathcal{E}(u))^{\frac{p_{*}-p}{p_{*}}} \\
& \text { or } \mathcal{E}_{p} \leq \mathcal{E}(u)
\end{aligned}
$$

Hence the proposition holds.

We have also:

Proposition 1.3. Let $u \in \dot{W}^{1, p}\left(\mathbb{R}^{N}\right) \backslash\{0\}$ be such that $\mathcal{E}(u)<\mathcal{E}_{p}$. Then we have

$$
\mathcal{K}(u) \neq 0, \quad\|u\|_{p_{*}} \neq\left\|w_{p}\right\|_{p_{*}} \text { and }\|\nabla u\|_{p} \neq\left\|\nabla w_{p}\right\|_{p}
$$

Moreover,

$$
\begin{aligned}
\mathcal{K}(u)<0 \Leftrightarrow\|u\|_{p_{*}}>\left\|w_{p}\right\|_{p_{*}} & \Leftrightarrow\|\nabla u\|_{p}>\left\|\nabla w_{p}\right\|_{p} . \\
\mathcal{K}(u)>0 \Leftrightarrow\|u\|_{p_{*}}<\left\|w_{p}\right\|_{p_{*}} & \Leftrightarrow\|\nabla u\|_{p}<\left\|\nabla w_{p}\right\|_{p} .
\end{aligned}
$$

Proof. $i) \bullet$ If we have $\mathcal{K}(u)=0$, then from (1.9) we have $\mathcal{E}_{p} \leq \mathcal{E}(u)$, which contradicts the hypothesis.

- Assume that $\|u\|_{p_{*}}=\left\|w_{p}\right\|_{p_{*}}$. Then we have by (1.10)

$$
\begin{aligned}
\mathcal{E}_{p} & =\frac{1}{p} \int_{\mathbb{R}^{N}}\left|\nabla w_{p}\right|^{p} d x-\frac{1}{p_{*}} \int_{\mathbb{R}^{N}}\left|w_{p}\right|^{p_{*}} d x \\
& =\frac{1}{p} \int_{\mathbb{R}^{N}}\left|\nabla w_{p}\right|^{p} d x-\frac{1}{p_{*}} \int_{\mathbb{R}^{N}}|u|^{p_{*}} d x \\
& \leq \frac{1}{p} \int_{\mathbb{R}^{N}}|\nabla u|^{p} d x-\frac{1}{p_{*}} \int_{\mathbb{R}^{N}}|u|^{p_{*}} d x \\
& =\mathcal{E}(u) .
\end{aligned}
$$

which again contradicts the hypothesis.

- Similarly, assume that $\|\nabla u\|_{p}=\left\|\nabla w_{p}\right\|_{p}$. Then arguing as before and using (1.11), we get a contradiction.

$i i) \bullet$ Assume that $\|u\|_{p_{*}}^{p_{*}}>\left\|w_{p}\right\|_{p_{*}}^{p_{*}}$. We recall from (1.15) that

$$
\begin{aligned}
\mathcal{E}(u) & =\frac{1}{p} \int_{\mathbb{R}^{N}}|\nabla u|^{p} d x-\frac{1}{p_{*}} \int_{\mathbb{R}^{N}}|u|^{p_{*}} d x \\
& =\frac{1}{p} \mathcal{K}(u)+\left(\frac{1}{p}-\frac{1}{p_{*}}\right) \int_{\mathbb{R}^{N}}|u|^{p_{*}} d x \\
& =\frac{1}{p} \mathcal{K}(u)+\frac{1}{N}\|u\|_{p_{*}}^{p_{*}} .
\end{aligned}
$$


Using the fact that $\mathcal{E}(u)<\mathcal{E}_{p}$ and (1.6)-(1.7), we obtain from (1.19)

$$
\begin{aligned}
\mathcal{K}(u) & =p \mathcal{E}(u)-\frac{p}{N}\|u\|_{p_{*}}^{p_{*}} \\
& <p \mathcal{E}_{p}-\frac{p}{N}\|u\|_{p_{*}}^{p_{*}} \\
& =\frac{p}{N}\left(\left\|w_{p}\right\|_{p_{*}}^{p_{*}}-\|u\|_{p_{*}}^{p_{*}}\right) \\
& <0 .
\end{aligned}
$$

- Assume that $\mathcal{K}(u)<0$. Then we have

$$
\int_{\mathbb{R}^{N}}|\nabla u|^{p} d x<\int_{\mathbb{R}^{N}}|u|^{p_{*}} d x
$$

Set $\widetilde{u}=\frac{\left\|w_{p}\right\|_{p_{*}}}{\|u\|_{p_{*}}} u$. Then clearly $\|\widetilde{u}\|_{p_{*}}=\left\|w_{p}\right\|_{p_{*}}$ and using (1.10), we have

$$
\begin{aligned}
& \int_{\mathbb{R}^{N}}\left|\nabla w_{p}\right|^{p} d x \leq \int_{\mathbb{R}^{N}}|\nabla \widetilde{u}|^{p} d x \\
& \text { that is } \int_{\mathbb{R}^{N}}\left|\nabla w_{p}\right|^{p} d x \leq \frac{\left\|w_{p}\right\|_{p_{*}}^{p}}{\|u\|_{p_{*}}^{p}} \int_{\mathbb{R}^{N}}|\nabla u|^{p} d x \\
& \text { or equivalently } \frac{1}{\left\|w_{p}\right\|_{p_{*}}^{p}} \int_{\mathbb{R}^{N}}\left|\nabla w_{p}\right|^{p} d x \leq \frac{1}{\|u\|_{p_{*}}^{p}} \int_{\mathbb{R}^{N}}|\nabla u|^{p} d x .
\end{aligned}
$$

Taking into account (1.6) and (1.20), we get from (1.21)

$$
\begin{aligned}
& \frac{\left\|w_{p}\right\|_{p_{*}}^{p_{*}}}{\left\|w_{p}\right\|_{p_{*}}^{p}}<\frac{\left.\|u\|\right|_{p_{*}} ^{p_{*}}}{\|u\|_{p_{*}}^{p}} \\
& \text { or }\left\|w_{p}\right\|_{p_{*}}^{p_{*}-p}<\|u\|_{p_{*}}^{p_{*}-p} \\
& \text { or }\left\|w_{p}\right\|_{p_{*}}<\|u\|_{p_{*}} .
\end{aligned}
$$

Thus $K(u)<0 \Leftrightarrow\|u\|_{p_{*}}>\left\|w_{p}\right\|_{p_{*}}$.

- Assume that $\mathcal{K}(u)<0$. We would like to prove that $\|\nabla u\|_{p}>\left\|\nabla w_{p}\right\|_{p}$. From the previous result and (1.4) we have

$$
\begin{aligned}
& \left\|w_{p}\right\|_{p_{*}}<\|u\|_{p_{*}} \leq C(N, p)\|\nabla u\|_{p} \\
& \text { or } \frac{1}{C(N, p)}\left\|w_{p}\right\|_{p_{*}}<\|\nabla u\|_{p}
\end{aligned}
$$

Taking into account (1.6), this leads to

$$
\begin{aligned}
& \frac{1}{C(N, p)}\left\|w_{p}\right\|_{p_{*}}=\left\|w_{p}\right\|_{p_{*}}^{1+\frac{p_{*}}{N}}<\|\nabla u\|_{p} \\
& \text { that is }\left\|w_{p}\right\|_{p_{*}}^{\frac{p_{*}}{p}}<\|\nabla u\|_{p} \\
& \text { or equivalently }\left\|\nabla w_{p}\right\|_{p}<\|\nabla u\|_{p} .
\end{aligned}
$$

- Conversely, assume that $\|\nabla u\|_{p}>\left\|\nabla w_{p}\right\|_{p}$ and let us show that necessarily $\mathcal{K}(u)<0$. Write

$$
\mathcal{K}(u)=p_{*}\left(\frac{1}{p}\|\nabla u\|_{p}^{p}-\frac{1}{p_{*}}\|u\|_{p_{*}}\right)-\frac{p}{N-p}\|\nabla u\|_{p}^{p} .
$$


From $\mathcal{E}(u)<\mathcal{E}\left(w_{p}\right)=\frac{1}{N}\left\|\nabla w_{p}\right\|_{p}^{p}$, we have

$$
\mathcal{K}(u)<p_{*} \mathcal{E}_{p}\left(w_{p}\right)-\frac{p}{N-p}\|\nabla u\|_{p}^{p}=\frac{p}{N-p}\left(\left\|\nabla w_{p}\right\|_{p}^{p}-\|\nabla u\|_{p}^{p}\right)<0
$$

as desired.

iii) The assertion (1.18) about $\mathcal{K}(u)>0$ is then a consequence of the previous two statements in Proposition 1.3.

\section{Blow-up in finite time of solutions}

The main result of this section is the following theorem.

Theorem 2.1. (Finite-time blow-up) Let $\left(u_{0}, u_{1}\right) \in\left(\dot{W}^{1, p}\left(\mathbb{R}^{N}\right) \cap L^{2}\left(\mathbb{R}^{N}\right)\right) \times L^{p}\left(\mathbb{R}^{N}\right)$. Assume that there exists a solution $u \in C^{0}\left(I, \dot{W}^{1, p}\left(\mathbb{R}^{N}\right)\right) \cap C^{1}\left(I, L^{p}\left(\mathbb{R}^{N}\right)\right)$ of (1.1) corresponding to the initial data $\left(u_{0}, u_{1}\right)$ defined on the maximal time interval $I$ and satisfying

$$
E\left(\left(u_{0}, u_{1}\right)\right)<E\left(\left(w_{p}, 0\right)\right) \text { and } \mathcal{K}\left(u_{0}\right)<0 .
$$

Then I must be a finite interval.

Remark 2.2. We point out that a blow-up result for the equation (1.1) has been obtained by Galaktinov-Pohozaev [4] under the following assumptions: $\int_{\mathbb{R}^{N}} u_{0} u_{1} d x>0$ and $E\left(\left(u_{0}, u_{1}\right)\right) \leq 0$, which are clearly more restrictive than our assumptions.

Remark 2.3. The existence of local solutions of (1.1) is still an interesting open question. The Cauchy problem is not only quasilinear but also with a degenerate or singular p-Laplacian ( $p>2$ or $1<p<2$, respectively). If a strong enough dissipation is put into the equation, then there are results about local or global existence of solution. See for example [5], [3] and [11].

In our proof, we follow an idea from [8] where the problem (1.1) was studied when $p=2$. To pave the way for the proof of Theorem 2.1, we shall need a number of lemmas.

Lemma 2.4. Let $u \in \dot{W}^{1, p}\left(\mathbb{R}^{N}\right)$ be such that we have, for some $\delta_{0}>0$,

$$
\|\nabla u\|_{p}^{p}<\left\|\nabla w_{p}\right\|_{p}^{p} \text { and } \mathcal{E}(u) \leq\left(1-\delta_{0}\right) \mathcal{E}_{p} .
$$

Then we have

$$
\|\nabla u\|_{p}^{p} \leq\left(1-\delta_{0}\right)\left\|\nabla w_{p}\right\|_{p}^{p} \text { and } \mathcal{E}(u)>0
$$

Proof. We argue as in the proof of [7] Lemma 3.4, and consider the function $f(y)=\frac{1}{p} y-\frac{C^{p_{*}}(N, p)}{p_{*}} y^{\frac{p_{*}}{p}}$ and set $\bar{y}=\|\nabla u\|_{p}^{p}$. We first observe that by the inequality (1.4)

$$
\begin{aligned}
f(\bar{y}) & =\frac{1}{p} \int_{\mathbb{R}^{N}}|\nabla u|^{p} d x-\frac{C^{p_{*}}(N, p)}{p_{*}}\left(\int_{\mathbb{R}^{N}}|\nabla u|^{p} d x\right)^{\frac{p_{*}}{p}} \\
& \leq \frac{1}{p} \int_{\mathbb{R}^{N}}|\nabla u|^{p} d x-\frac{C^{p_{*}}(N, p)}{p_{*}} \frac{1}{C^{p_{*}}(N, p)} \int_{\mathbb{R}^{N}}|u|^{p_{*}} d x \\
& =\mathcal{E}(u) \\
& \leq\left(1-\delta_{0}\right) \mathcal{E}_{p} \\
& =\frac{1-\delta_{0}}{N C^{N}(N, p)} .
\end{aligned}
$$


Next we have

$$
\begin{aligned}
& f(0)=0 \\
& f^{\prime}(y)= \frac{1}{p}-\frac{C^{p_{*}}(N, p)}{p_{*}} \frac{p_{*}}{p} y^{\frac{p_{*}}{p}-1}=\frac{1}{p}-\frac{C^{p_{*}}(N, p)}{p} y^{\frac{p}{N-p}} \\
& f^{\prime \prime}(y)=-\frac{C^{p_{*}}(N, p)}{N-p} y^{\frac{2 p-N}{N-p}} \leq 0 \\
& f^{\prime}(y)=0 \Leftrightarrow y=y_{C}=\frac{1}{C^{N}(N, p)}=\left\|\nabla w_{p}\right\|_{p}^{p} \\
& f\left(y_{C}\right)=\frac{1}{p} y_{C}-\frac{C^{p_{*}}(N, p)}{p_{*}} y_{C}^{\frac{p_{*}}{p}} \\
&=\frac{1}{p} \frac{1}{C^{N}(N, p)}-\frac{C^{p_{*}}(N, p)}{p_{*}} \frac{1}{(C(N, p))^{\frac{N^{2}}{N-p}}} \\
&=\frac{1}{p} \frac{1}{C^{N}(N, p)}-\frac{1}{p_{*}} \frac{1}{C^{N}(N, p)} \\
&=\frac{1}{N C^{N}(N, p)} .
\end{aligned}
$$

It follows that $f$ is nonnegative and strictly increasing between 0 and $y_{C}$ and concave in $[0, \underline{y}]$, where $\underline{y}=$ $\frac{1}{C^{N}(N, p)}\left(\frac{N}{N-p}\right)^{\frac{N-p}{p}}$ is such that $f(\underline{y})=0$.

Since $f$ is strictly increasing between 0 and $y_{C}$ and $0<\bar{y}<y_{C}$, we obtain from (2.1) $\mathcal{E}(u) \geq f(\bar{y})>0$.

Using the concavity of $f$ and (2.1), we obtain

$$
f(\bar{y}) \leq\left(1-\delta_{0}\right) f\left(y_{C}\right) \leq f\left(\left(1-\delta_{0}\right) y_{C}\right) .
$$

Since $f$ is strictly increasing between 0 and $y_{C}$, we obtain from (2.2)

$$
\bar{y} \leq\left(1-\delta_{0}\right) y_{C} \Leftrightarrow\|\nabla u\|_{p}^{p} \leq\left(1-\delta_{0}\right)\left\|\nabla w_{p}\right\|_{p}^{p} .
$$

Lemma 2.5. Let $u \in \dot{W}^{1, p}\left(\mathbb{R}^{N}\right)$ be such that we have, for some $\delta_{0}>0$,

$$
\mathcal{K}(u)<0 \text { and } \mathcal{E}(u) \leq\left(1-\delta_{0}\right) \mathcal{E}_{p}
$$

Then we have for the positive constant $\bar{\delta}=\left(1+\frac{p \delta_{0}}{N-p}\right)^{\frac{N-p}{p}}-1$

$$
\|\nabla u\|_{p}^{p} \geq(1+\bar{\delta})\left\|\nabla w_{p}\right\|_{p}^{p}
$$

Proof. From the assumption and Proposition 1.3, we know that $\|\nabla u\|_{p}>\left\|\nabla w_{p}\right\|_{p}$. Using the inequality (1.4) and (1.6)-(1.7), we obtain

$$
\begin{aligned}
\|\nabla u\|_{p}^{p} & >\left\|\nabla w_{p}\right\|_{p}^{p}=N \mathcal{E}_{p} \geq \frac{N}{1-\delta_{0}} \mathcal{E}(u) \\
& \geq \frac{N}{1-\delta_{0}}\left(\frac{1}{p} \int_{\mathbb{R}^{N}}|\nabla u|^{p} d x-\frac{C^{p_{*}}(N, p)}{p_{*}}\left(\int_{\mathbb{R}^{N}}|\nabla u|^{p} d x\right)^{\frac{p_{*}}{p}}\right) \\
& =\frac{N}{1-\delta_{0}}\left(\frac{1}{p} \int_{\mathbb{R}^{N}}|\nabla u|^{p} d x-\frac{C^{p_{*}}(N, p)}{p_{*}}\left(\int_{\mathbb{R}^{N}}|\nabla u|^{p} d x\right)^{\frac{N}{N-p}}\right) .
\end{aligned}
$$


This leads to

$$
\begin{aligned}
& \frac{N C^{p_{*}}(N, p)}{\left(1-\delta_{0}\right) p_{*}}\left(\int_{\mathbb{R}^{N}}|\nabla u|^{p} d x\right)^{\frac{p}{N-p}} \geq \frac{N}{\left(1-\delta_{0}\right) p}-1=\frac{N-p+p \delta_{0}}{\left(1-\delta_{0}\right) p} \\
& \text { or }\left(\int_{\mathbb{R}^{N}}|\nabla u|^{p} d x\right)^{\frac{p}{N-p}} \geq \frac{N-p+p \delta_{0}}{N-p} \frac{1}{C^{p_{*}}(N, p)} \\
& \text { or } \int_{\mathbb{R}^{N}}|\nabla u|^{p} d x \geq\left(1+\frac{p \delta_{0}}{N-p}\right)^{\frac{N-p}{p}} \frac{1}{C^{N}(N, p)}=(1+\bar{\delta})\left\|\nabla w_{p}\right\|_{p}^{p}
\end{aligned}
$$

and the lemma follows.

Lemma 2.6. Let $\left(u_{0}, u_{1}\right) \in \dot{W}^{1, p}\left(\mathbb{R}^{N}\right) \times L^{2}\left(\mathbb{R}^{N}\right)$. Assume that there exists a solution $u$ of $(1.1)$ corresponding to the initial data $\left(u_{0}, u_{1}\right)$ defined on the maximal interval I and satisfying $E\left(\left(u_{0}, u_{1}\right)\right)<E\left(\left(w_{p}, 0\right)\right)$. Then we have

$$
\mathcal{K}\left(u_{0}\right)<0 \Rightarrow\|\nabla u(t)\|_{p}>\left\|\nabla w_{p}\right\|_{p} \quad \forall t \in I .
$$

Proof. Let $\delta_{0}>0$ be a fixed positive number such that $E\left(\left(u_{0}, u_{1}\right)\right)<\left(1-\delta_{0}\right) E\left(\left(w_{p}, 0\right)\right)$. Since $\mathcal{E}\left(u_{0}\right) \leq$ $E\left(\left(u_{0}, u_{1}\right)\right) \leq\left(1-\delta_{0}\right) E\left(\left(w_{p}, 0\right)\right)=\left(1-\delta_{0}\right) \mathcal{E}_{p}$ and $\mathcal{K}\left(u_{0}\right)<0$, we know from Proposition 1.3 that

$$
\left\|\nabla u_{0}\right\|_{p}>\left\|\nabla w_{p}\right\|_{p}
$$

Now let $t \in I$. By (1.2), we have $E\left(\left(u(t), u_{t}(t)\right)\right)=E\left(\left(u_{0}, u_{1}\right)\right)$. We deduce that

$$
\mathcal{E}(u(t)) \leq E\left(\left(u(t), u_{t}(t)\right)\right)=E\left(\left(u_{0}, u_{1}\right)\right) \leq\left(1-\delta_{0}\right) E\left(\left(w_{p}, 0\right)\right)=\left(1-\delta_{0}\right) \mathcal{E}_{p} .
$$

If $\left\|\left.\nabla u(t)\right|_{p} ^{p}<\right\| \nabla w_{p} \|_{p}^{p}$, we obtain from (2.5) and Lemma 2.4

$$
\|\nabla u(t)\|_{p}^{p} \leq\left(1-\delta_{0}\right)\left\|\nabla w_{p}\right\|_{p}^{p}
$$

If $\|\nabla u(t)\|_{p}^{p}>\left\|\nabla w_{p}\right\|_{p}^{p}$, we have by Lemma 2.5 , for $\bar{\delta}=\left(1+\frac{p \delta_{0}}{N-p}\right)^{\frac{N-p}{p}}-1$

$$
\|\nabla u(t)\|_{p}^{p} \geq(1+\bar{\delta})\left\|\nabla w_{p}\right\|_{p}^{p}
$$

Taking into account (2.4) and the continuity of $\|\nabla u(t)\|_{p}$, the lemma holds.

Remark 2.7. Under the assumption of Lemma 2.6, we can similarly show that

$$
\left\|\nabla u_{0}\right\|_{p}<\left\|\nabla w_{p}\right\|_{p} \Rightarrow\|\nabla u(t)\|_{p}<\left\|\nabla w_{p}\right\|_{p} \quad \forall t \in I .
$$

We are now able to prove the main result of the paper.

Proof. of Theorem 2.1. Let $\delta_{0}>0$ be a fixed positive number such that

$$
E\left(\left(u_{0}, u_{1}\right)\right)<\left(1-\delta_{0}\right) E\left(\left(w_{p}, 0\right)\right)
$$


and let $y(t)=\int_{\mathbb{R}^{N}}|u(x, t)|^{2} d x$. We obtain by using (1.1) and integrating by parts

$$
\begin{aligned}
y^{\prime}(t) & =2 \int_{\mathbb{R}^{N}} u(x, t) u_{t}(x, t) d x \\
y^{\prime \prime}(t) & =2 \int_{\mathbb{R}^{N}}\left|u_{t}(x, t)\right|^{2} d x+2 \int_{\mathbb{R}^{N}} u(x, t) u_{t t}(x, t) d x \\
& =2 \int_{\mathbb{R}^{N}}\left|u_{t}(x, t)\right|^{2} d x+2 \int_{\mathbb{R}^{N}} u\left(\Delta_{p} u+|u|^{p_{*}-2} u\right) d x \\
& =2 \int_{\mathbb{R}^{N}}\left|u_{t}(x, t)\right|^{2} d x-2 \mathcal{K}(u(t)) \\
& =2 \int_{\mathbb{R}^{N}}\left|u_{t}(x, t)\right|^{2} d x-2 \int_{\mathbb{R}^{N}}|\nabla u(t)|^{p} d x+2 \int_{\mathbb{R}^{N}}|u(x, t)|^{p_{*}} d x .
\end{aligned}
$$

For convenience, we set $\widetilde{\delta}_{0}=\delta_{0} E\left(\left(w_{p}, 0\right)\right)$, and we obtain

$$
E\left(\left(w_{p}, 0\right)\right) \geq E\left(\left(u_{0}, u_{1}\right)\right)+\widetilde{\delta}_{0}=E\left(\left(u(t), u_{t}(t)\right)\right)+\widetilde{\delta}_{0},
$$

which leads to

$$
\begin{aligned}
\int_{\mathbb{R}^{N}}|u(x, t)|^{p_{*}} d x \geq & \frac{p_{*}}{2} \int_{\mathbb{R}^{N}}\left|u_{t}(x, t)\right|^{2} d x+\frac{p_{*}}{p} \int_{\mathbb{R}^{N}}|\nabla u(t)|^{p} d x \\
& -p_{*} E\left(\left(w_{p}, 0\right)\right)+\widetilde{\delta}_{0} p_{*} .
\end{aligned}
$$

We deduce from (2.6)-(2.7) and Lemma 2.6 that

$$
\begin{aligned}
y^{\prime \prime}(t) \geq & \left(2+p_{*}\right) \int_{\mathbb{R}^{N}}\left|u_{t}(x, t)\right|^{2} d x+\frac{2 p_{*}}{p} \int_{\mathbb{R}^{N}}|\nabla u(t)|^{p} d x \\
& -2 p_{*} E\left(\left(w_{p}, 0\right)\right)+2 \widetilde{\delta}_{0} p_{*}-2 \int_{\mathbb{R}^{N}}|\nabla u(t)|^{p} d x \\
= & \left(2+p_{*}\right) \int_{\mathbb{R}^{N}}\left|u_{t}(x, t)\right|^{2} d x+2\left(\frac{p_{*}}{p}-1\right) \int_{\mathbb{R}^{N}}|\nabla u(t)|^{p} d x \\
& -\frac{2 p_{*}}{N} \int_{\mathbb{R}^{N}}\left|\nabla w_{p}\right|^{p} d x+2 \widetilde{\delta}_{0} p_{*} \\
= & \left(2+p_{*}\right) \int_{\mathbb{R}^{N}}\left|u_{t}(x, t)\right|^{2} d x+\frac{2 p_{*}}{N}\left(\int_{\mathbb{R}^{N}}|\nabla u(t)|^{p} d x-\int_{\mathbb{R}^{N}}\left|\nabla w_{p}\right|^{p} d x\right)+2 \widetilde{\delta}_{0} p_{*} \\
\geq & \left(2+p_{*}\right) \int_{\mathbb{R}^{N}}\left|u_{t}(x, t)\right|^{2} d x+2 \widetilde{\delta}_{0} p_{*} .
\end{aligned}
$$

Assuming that $[0, \infty) \subset I$, and integrating (2.8), we obtain for $t>0, y^{\prime}(t) \geq 2 \widetilde{\delta}_{0} p_{*} t+y^{\prime}(0)$. We deduce that there exists $t_{0}>0$ such that $y^{\prime}(t)>0$ for all $t \geq t_{0}$. We obtain for $t>t_{0}$, by using Cauchy-Schwarz inequality

$$
\begin{aligned}
& y^{\prime \prime}(t) y(t) \geq\left(2+p_{*}\right)\left(\int_{\mathbb{R}^{N}}\left|u_{t}(x, t)\right|^{2} d x\right)\left(\int_{\mathbb{R}^{N}}|u(x, t)|^{2} d x\right) \geq \frac{p_{*}+2}{4} y^{\prime 2}(t) \\
& \text { or } \frac{y^{\prime \prime}(t)}{y^{\prime}(t)} \geq \frac{p_{*}+2}{4} \frac{y^{\prime}(t)}{y(t)} .
\end{aligned}
$$

Integrating (2.9) between $t_{0}$ and $t$, we obtain for $C_{0}=\frac{y^{\prime}\left(t_{0}\right)}{\left(y\left(t_{0}\right)\right)^{\frac{p_{*}+2}{4}}}$

$$
\begin{aligned}
& \ln \left(y^{\prime}(t)\right) \geq \frac{p_{*}+2}{4} \ln (y(t))+\ln \left(C_{0}\right) \\
& \text { or } y^{\prime}(t) \geq C_{0}(y(t))^{\frac{p_{*}+2}{4}} \\
& \text { or } \frac{y^{\prime}(t)}{(y(t))^{\frac{p_{*}+2}{4}}} \geq C_{0} .
\end{aligned}
$$


Integrating again (2.10) between $t_{0}$ and $t$, we obtain for $C_{1}=\frac{4}{\left(p_{*}-2\right)\left(y\left(t_{0}\right)\right)^{\frac{p_{*}-2}{4}}}$

$$
\frac{4}{\left(p_{*}-2\right)(y(t))^{\frac{p_{*}-2}{4}}} \leq C_{0}\left(t_{0}-t\right)+C_{1} .
$$

We have reached a contradiction, since the right hand side of the last inequality becomes negative for $t$ large enough, while the left hand side is always nonnegative, since $p_{*}>2$.

In the same way we get a contradiction, if we assume that $(-\infty, 0] \subset I$

Remark 2.8. From the proof of the Theorem, we see that we just needed an energy inequality which might be satisfied by just weak solutions.

Remark 2.9. Define the sets:

$$
\begin{aligned}
& R_{1}=\left\{(u, v) \in \dot{W}^{1, p}\left(\mathbb{R}^{N}\right) \times L^{2}\left(\mathbb{R}^{N}\right): E((u, v))<\mathcal{E}_{p} \text { and } \mathcal{K}(u)>0\right\} \cup\{(0,0)\} \\
& R_{2}=\left\{(u, v) \in \dot{W}^{1, p}\left(\mathbb{R}^{N}\right) \times L^{2}\left(\mathbb{R}^{N}\right): E((u, v))<\mathcal{E}_{p} \text { and } \mathcal{K}(u)<0\right\} .
\end{aligned}
$$

It is clear from Proposition 1.3, Lemma 2.6 and Remark 2.7, that $R_{1}$ and $R_{2}$ are invariant sets under the flow of the wave equation (1.1). Moreover, Theorem 2.1 shows that data in $R_{2}$ lead to finite time blow up solutions. However, we were not able so far to show that data in $R_{1}$ lead to global solutions. Nevertheless, we have the following $\grave{a}$ priori bounds on the solutions: for all $t \in I$ the maximal time of existence

$$
\begin{gathered}
\int_{\mathbb{R}^{N}}|\nabla u(t)|^{p} d x<N \mathcal{E}_{p}, \quad \forall t \in I, \\
\frac{1}{2} \int_{\mathbb{R}^{N}}\left|u_{t}(t)\right|^{2} d x+\frac{1}{N} \int_{\mathbb{R}^{N}}|u(t)|^{p_{*}} d x<\mathcal{E}_{p} \quad \forall t \in I .
\end{gathered}
$$

Indeed, by assumption we have $\left(u_{0}, u_{1}\right) \in R_{1}$ and therefore we obtain from (1.2) and the continuity of $\mathcal{K}(u(t))$ that $\left(u(t), u_{t}(t)\right) \in R_{1}$ for all $t \in I$. In particular we have

$$
\mathcal{K}(u(t))>0 \quad \forall t \in I,
$$

and

which can be written

$$
E\left(\left(u(t), u_{t}(t)\right)\right)=E\left(\left(u_{0}, u_{1}\right)\right)<\mathcal{E}_{p}, \quad \forall t \in I
$$

$$
\begin{aligned}
& \frac{1}{2} \int_{\mathbb{R}^{N}}\left|u_{t}(t)\right|^{2} d x+\frac{1}{p} \int_{\mathbb{R}^{N}}|\nabla u(t)|^{p} d x-\frac{1}{p_{*}} \int_{\mathbb{R}^{N}}|u(t)|^{p_{*}} d x<\mathcal{E}_{p} \\
& \text { or } \frac{1}{2} \int_{\mathbb{R}^{N}}\left|u_{t}(t)\right|^{2} d x+\frac{1}{p} \mathcal{K}(u(t))+\frac{1}{N} \int_{\mathbb{R}^{N}}|u(t)|^{p_{*}} d x<\mathcal{E}_{p} .
\end{aligned}
$$

It follows from (2.11)-(2.12) that we have

$$
\frac{1}{2} \int_{\mathbb{R}^{N}}\left|u_{t}(t)\right|^{2} d x+\frac{1}{N} \int_{\mathbb{R}^{N}}|u(t)|^{p_{*}} d x<\mathcal{E}_{p} \forall t \in I .
$$

Now using (2.13), we obtain also from (2.12) that

$$
\int_{\mathbb{R}^{N}}|\nabla u(t)|^{p} d x<p \mathcal{E}_{p}+\frac{p}{p_{*}} \int_{\mathbb{R}^{N}}|u(t)|^{p_{*}} d x<\left(p+\frac{N p}{p_{*}}\right) \mathcal{E}_{p}=N \mathcal{E}_{p}, \quad \forall t \in I
$$

Acknowledgements. The second author would like to thank the Fields Institute for the facilities and excellent research conditions during his stay at this institution. This work was done during the "Workshop on wave breaking and global solutions" held at the Fields Institute, Toronto, Canada, May 2-6, 2011. 


\section{References}

[1] M. Agueh. A new ODE approach to sharp Sobolev inequalities. Nonlinear Analysis Research Trends. Nova Science Publishers, Inc. Editor: Inès N. Roux, pp. 1-13 (2008).

[2] T. Aubin. Problème isopérimétrique et espaces de Sobolev, J. Differential Geometry. 11, pp. 573-598 (1976).

[3] C. Chen, H. Yao, L. Shao. Global Existence, Uniqueness, and Asymptotic Behavior of Solution for p-Laplacian Type Wave Equation. Journal of Inequalities and Applications Volume 2010, Article ID 216760, 15 pages.

[4] V.A. Galaktionov, S.I. Pohozaev. Blow-up and critical exponents for nonlinear hyperbolic equations. Nonlinear Analysis 53, pp. 453-466 (2003).

[5] G. Hongjun, Z. Hui. Global nonexistence of the solutions for a nonlinear wave equation with the q-Laplacian operator. J. Partial Diff. Eqs. 20 pp. 71-79(2007).

[6] S. Ibrahim, N. Masmoudi, K. Nakanishi. Scattering threshold for the focusing nonlinear Klein-Gordon equation. Analysis and PDE 4, No. 3, pp. 405-460, 2011.

[7] C. E. Kenig, F. Merle. Global well-posedness, scattering and blow-up for the energy-critical, focusing, non-linear Schrödinger equation in the radial case. Invent. Math. 166, No. 3, pp. 645-675 (2006).

[8] C. E. Kenig, F. Merle. Global well-posedness, scattering and blow-up for the energy-critical focusing non-linear wave equation. Acta Math. 201, No. 2, pp. 147-212 (2008).

[9] J. Shatah. Unstable ground state of nonlinear Klein-Gordon equations. Trans. Amer. Math. Soc. 290, No. 2, pp. $701-710$ (1985).

[10] G. Talenti. Best constants in Sobolev inequality. Ann. Mat. Pura Appl. 110, pp. 353-372 (1976).

[11] Z. Wilstein. Global Well-Posedness for a Nonlinear Wave Equation with p-Laplacian Damping. Ph.D. thesis, University of Nebraska. http://digitalcommons.unl.edu/mathstudent/24 